\title{
COSPAN CONSTRUCTION OF THE GRAPH CATEGORY OF BORISOV AND MANIN
}

\author{
JOACHIM KOCK
}

To Nils Baas, on his 70th birthday

\begin{abstract}
It is shown how the graph category of Borisov and Manin can be constructed from (a variant of) the graph category of Joyal and Kock, essentially by reversing the generic morphisms. More precisely, the morphisms in the Borisov-Manin category are exhibited as cospans of reduced covers and refinement morphisms.
\end{abstract}

2010 Mathematics Subject Classification: 18D50, 05C99.

Key words: Graphs, generalised operads.

\section{Introduction}

The following definition of graph with open-ended edges is quite standard in the literature, and is usually attributed to Kontsevich and Manin [13]: a graph is a quadruple $(V, F, \partial, j)$ where $V$ is a set of vertices, $F$ is a set of flags (also called half-edges), $\partial: F \rightarrow V$ is a map (assigning to a flag the vertex it originates from), and $j: F \rightarrow F$ is an involution. The idea is that the involution interchanges two flags if they are attached to each other to form an edge. The fixpoints for $j$ are interpreted as open-ended edges, called tails (this set is denoted $T$ ).

The 'problem' with this definition (due to the fact that tails are encoded as fixpoints, although a tail is not a flag glued to itself) is that it does not naturally lead to good notions of morphisms, beyond isomorphisms: naive attempts in terms of structure-preserving maps tend to preserve too much structure.

Borisov and Manin [4] overcame this problem with a rather intricate definition of morphism, reproduced below. Their category is rich enough to capture the combinatorics of both operad-like and prop-like structures.

A main point to make to explain the significance of the notion and the intuition behind it is that there are two main classes of morphisms, called graftings and compressions (the compressions are spanned by the 
contractions and mergers of Borisov-Manin), both expressing operations that are fundamental to graph theory: a grafting is a morphism that takes a graph (connected or not) and constructs a new graph by clutching together some of its tails to form edges. A compression is a morphism that takes a graph, chooses a collection of nonempty (but not necessarily connected) subgraphs, and contracts each of them to a vertex. The point of the Borisov-Manin category is that every morphism factors as a grafting followed by a compression, and that this factorisation is essentially unique (cf. Proposition 2.6 below). In this way their category can be seen to be the smallest category containing these two classes of morphisms and a sensible notion of composition of such morphisms. It is remarkable that an elementary description could be found to achieve this.

The present contribution takes the factorisation property as the starting point for a description of the category, instead of the intricate elementary description. This can be formalised through a general procedure for constructing a category from two given classes of morphisms, $G$ and $C$, with a common object set. The new morphisms are declared to be formal pairs consisting of a morphism in $G$ followed by a morphism in $C$. To define the composition law for such pairs, one needs a rule for transforming a pair in the wrong order into a pair in the right order, subject to some axioms reminiscent of the axioms for bimodules or braids. This is called a commutation law, or sometimes a distributive law [16].

In this strategy, the question then becomes first to describe the two classes of morphisms individually, hopefully in a more conceptual way, and then to specify the commutation law. Most of this has already been accomplished, namely in the Joyal-Kock formalism of graphs [9], recalled below. This formalism starts with a definition of graph featuring a canonical notion of etale morphism (such as open inclusions). This defines the etale topology and a notion of etale cover; a cover is reduced when it is bijective on vertices. A reduced cover is essentially the same thing as a grafting in the sense of Borisov-Manin (cf. Proposition 3.6).

The second class of morphisms is generated from the first by a monad, a version of the free-compact-symmetric-multicategory monad [9], which could be called the free-compact-coloured-prop monad: this is the class of generic morphisms, a general categorical notion [17], which in this case can be described in elementary terms as refinements. A refinement is the opposite of a compression (Proposition 5.4). Note here that since a compression is allowed to contract a non-connected graph, an important 
minor adjustment is required in the description of the monad compared to $[\mathbf{9}]$ (essentially passing from connected to non-connected).

These two classes of morphisms already live in a single bigger category, namely the Kleisli category for the monad $[\mathbf{9}]$, but in here they interact differently since a refinement morphism between two graphs in the Kleisli category goes in the opposite direction of the corresponding compression morphism in the BM category. However, the key observation at this point is that reduced covers and refinement morphisms admit pushouts along each other, yielding new morphisms in the same classes (Proposition 6.1). The pushout construction is precisely the required commutation law - in this case a prescription for formally reversing one of the two classes: the new morphisms from graph $\mathrm{T}$ to graph $\mathrm{R}$ are the cospans in which the first leg is a reduced cover and the second leg a refinement morphism:

$$
\mathrm{T} \stackrel{\text { red.cover }}{\longrightarrow} \mathrm{S} \stackrel{\text { refinement }}{\longleftarrow} \mathrm{R} \text {. }
$$

The composition of cospans is given by pushout. Now the main result is this:

Theorem 6.6. The Borisov-Manin category of graphs is equivalent to the category whose morphisms are cospans of reduced covers and refinement morphisms in the Kleisli category.

The result shows that the Borisov-Manin category of graphs can be derived from general principles and standard constructions: indeed the notions of etale morphism and reduced cover are completely canonical, and the notion of generic morphism is characterised by a universal property, relative to the monad, in turn defined in terms of colimits and presheaves. This gives some theoretical justification for the BM definition, complementing the significant empirical fact that it just works, and complementing its pleasing elementary character.

Although the main arguments in the proof are conceptual, some details of the comparison must be done by hand. These calculations are illustrative for both formalisms, and it is hoped that they too can be of some interest also beyond the theorem established.

\section{Borisov-Manin category of graphs}

With graphs defined as in the first paragraph of the introduction, the graph category $\boldsymbol{B M}$ of Borisov and Manin [4] has the following morphisms. 
Definition $([4,1.2 .1])$. Let $\tau, \sigma$ be two graphs. A morphism $h: \tau \rightarrow \sigma$ is a triple $\left(h^{F}, h_{V}, j_{h}\right)$, where $h^{F}: F_{\sigma} \rightarrow F_{\tau}$ is a contravariant map, $h_{V}: V_{\tau} \rightarrow V_{\sigma}$ is a covariant map, and $j_{h}$ is an involution on the set $F_{\tau} \backslash h^{F}\left(F_{\sigma}\right)$. This data must satisfy the following conditions.

(i) $h^{F}$ is injective, $h_{V}$ is surjective.

(ii) The image $h^{F}\left(F_{\sigma}\right)$ and its complement $F_{\tau} \backslash h^{F}\left(F_{\sigma}\right)$ are $j_{\tau}$-invariant subsets of flags.

(ii') The involution $j_{h}$ is fixpoint free, and agrees with $j_{\tau}$ on edges (but must necessarily disagree on tails, since $j_{h}$ is fixpoint free).

We will say that $h$ contracts all flags in $F_{\tau} \backslash h^{F}\left(F_{\sigma}\right)$. If two flags in $F_{\tau} \backslash h^{F}\left(F_{\sigma}\right)$ form an edge, we say that this edge is contracted by $h$. If two tails in $F_{\tau} \backslash h^{F}\left(F_{\sigma}\right)$ form an orbit of $j_{h}$, we say that it is a virtual edge contracted by $h$.

(iii) If a flag $f_{\tau}$ is not contracted by $h$, that is, has the form $h^{F}\left(f_{\sigma}\right)$, then $h_{V}$ sends $\partial_{\tau} f_{\tau}$ to $\partial_{\sigma} f_{\sigma}$. Two vertices of a contracted edge (actual or virtual) must have the same $h_{V}$-image.

(iv) The bijection $h_{F}^{-1}: h^{F}\left(F_{\sigma}\right) \rightarrow F_{\sigma}$ maps edges of $\tau$ to edges of $\sigma$.

If it maps a pair of tails of $\tau$ to an edge of $\sigma$, we will say that $h$ grafts these tails.

Given two morphisms

$$
\tau \stackrel{g}{\longrightarrow} \sigma \stackrel{c}{\longrightarrow} \rho
$$

the composite morphism $h=c \circ g$ is defined to have $h_{V}=c_{V} \circ g_{V}$ and $h^{F}=g^{F} \circ c^{F}$. To complete the definition of $h$ we must define the fixpoint-free involution $j_{h}$ on the complement of $h^{F}$, which is the (disjoint) union of the complement of $g^{F}$ in $F_{\tau}$ and the complement of $c^{F}$ in $F_{\sigma} \subset F_{\tau}$. We define $j_{h}$ to be $j_{g}$ on the first and $j_{c}$ on the second.

The definition is reproduced almost verbatim from [4], only with a slight adjustment to the definition of $j_{h}$, here defined on the whole complement. In [4] it is defined only on the set of tails in the complement. This adjustment facilitates the description of the composition law (adjusted accordingly).

2.1. Grafting. A morphism of graphs $h: \tau \rightarrow \sigma$ is called a grafting when both $h_{V}$ and $h^{F}$ are bijective.

2.2. Compression. A morphism of graphs $h: \tau \rightarrow \sigma$ is called a compression when $h^{F}$ is bijective on tails. 
The notion of grafting is verbatim from [4]. The class of compressions is spanned by two classes of morphisms described in [4]: contractions and mergers. A contraction is a compression for which any two vertices with the same image under $h_{V}$ are connected by a path of contracted edges in $\tau$. A merger is a compression for which $h^{F}$ is also bijective on edges. The reason for singling out the mergers is that in many applications, this class of morphisms is actually excluded. It is excluded in operad-like situations (operads, properads, cyclic operads, modular operads, etc.), whereas it is needed in prop-like situations (props, wheeled props, etc.). The reason presently for treating the two classes together is Proposition 2.6 below.

Lemma 2.3. A grafting or compression $h$ is uniquely determined by $h_{V}$ and $h^{F}$ (i.e. $j_{h}$ is implied).

Proof: Indeed, for a grafting, $h^{F}$ is bijective, so there is no complement to define $j_{h}$ on. For a compression, the complement consists entirely of edges (since $h^{F}$ is a bijection on tails), and here we are forced to let $j_{h}$ agree with $j_{\tau}$ (by Axiom (ii')).

Lemma 2.4. For a compression $h: \sigma \rightarrow \rho$, the flag map $h^{F}: F_{\rho} \rightarrow F_{\sigma}$ sends edges to edges, tails to tails, and in particular commutes with the involutions $j_{\rho}$ and $j_{\sigma}$.

Proof: Since $h^{F}$ induces a bijection on tails, it must also map nontails to non-tails. On the other hand, by Axiom (iv) the inverse map $\left(h^{F}\right)^{-1}: h^{F}\left(F_{\rho}\right) \rightarrow F_{\rho}$ maps edges to edges, so it follows that $h^{F}$ maps edges to edges. Thus altogether it maps tails to tails and edges to edges, and hence commutes with the involutions.

The following lemma expresses the important property that the 'subgraph' compressed by a compression has the same 'interface' (to be formalised in 3.1) as the vertex it is compressed to. The formulation is slightly awkward here due to the fact that the notion of subgraph has not been defined (and at any rate is not given by a morphism in the category):

Lemma 2.5. For a compression $h: \sigma \rightarrow \rho$, and for each vertex $x \in V_{\rho}$, the flag map $h^{F}: F_{\rho} \rightarrow F_{\sigma}$ restricts to a bijection

$$
\partial^{-1}(x) \stackrel{\sim}{\longrightarrow} h^{F}\left(F_{\rho}\right) \cap \partial^{-1} h_{V}^{-1}(x) .
$$

Proof: Injectivity follows from Axiom (i). For surjectivity, note that a $\sigma$-flag on the right-hand side must come from some $\rho$-flag, and now it follows from Axiom (iii) that this flag is incident to $x$. 
Proposition 2.6. Every morphism of graphs $h: \tau \rightarrow \rho$ factors essentially uniquely as

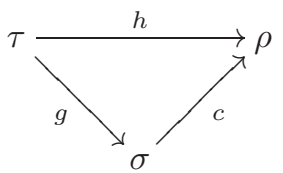

where $g$ is a grafting and $c$ is a compression. More precisely, the classes of graftings and compressions form a factorisation system in the BorisovManin category of graphs.

Proof: We construct the middle object $\sigma$ in the factorisation. Since we want a grafting $\tau \rightarrow \sigma$, we are forced to take as vertices and flags of $\sigma$ the same as those of $\tau$. Since now $g_{V}$ and $g^{F}$ are bijections, this also determines $c_{V}:=h_{V} \circ g_{V}^{-1}$ and $c^{F}:=\left(g^{F}\right)^{-1} \circ h^{F}$. To specify $\sigma$, it only remains to choose the involution $j_{\sigma}$. By Lemma $2.4, j_{\sigma}$ must agree with $j_{\rho}$ on the image of $c^{F}$ (which is also the image of $h^{F}$ ). Now we have to define $j_{\sigma}$ outside the image of $c^{F}$. Whatever we choose, this assignment will define also $j_{c}$ (by Lemma 2.3). But the composite of $g$ and $c$ will have $j_{c g}$ equal to $j_{c}$ (since $g^{F}$ has empty complement). So in order to have $c \circ g=h$, we need $j_{c}=j_{h}$, so we are forced to take $j_{\sigma}=j_{h}$. Now we have constructed a factorisation. For any two possible factorisations (which may differ only by the names of the elements in the sets), there is a unique comparison, determined by the bijections already involved. Hence the factorisation is essentially unique. We have established that the two classes of morphisms constitute a factorisation system.

2.7. Commutation law. We shall need the special case of factorisation where a composable pair of arrows in the 'wrong order' is composed and then factorised into a pair in the 'right order'. This interchange of order is an example of the general notion of commutation law. The axioms for a commutation law will not be listed, since they are automatically satisfied for commutation laws given by a factorisation system, as is the case here (and in Proposition 6.5). Given a compression morphism $h: \tau \rightarrow \omega$ and a grafting $k: \omega \rightarrow \rho$,

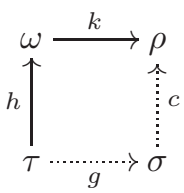

the commutation law completes the square with $g$ a grafting and $c$ a compression. As in the proof above, the vertices and flags of $\sigma$ are those 
of $\tau$, with $g_{V}$ and $g^{F}$ the identity maps. Only the involution $j_{\sigma}$ changes: $g$ grafts the 'same' tails as $k$ does - this makes sense since $h$ is bijective on tails. The compression $c$ has $c_{V}=h_{V}$ and $c^{F}=h^{F}$, modulo the bijections that identity vertices and flags of $\omega$ with vertices and flags of $\rho$. It other words, it compresses the same 'subgraphs' as $h$ does.

\section{Joyal-Kock category of graphs and etale morphisms}

The following definition of graph is from Joyal-Kock [9]. The definition can be seen as an natural open-ended version of the Serre definition of graph, and it is also in the spirit of the polynomial tree formalism in [11]. A directed variant with the same features was given in [12].

3.1. Definition of graph. A graph is a diagram of finite sets

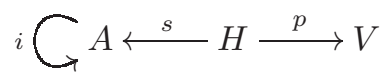

such that $s$ is injective and $i$ is a fixpoint-free involution.

The set $V$ is the set of vertices. The set $H$ is the set of half-edges or flags: these are pairs consisting of a vertex together with the germ of an emanating arc. Finally the set $A$ is the set of arcs, which can be thought of as an edge with a chosen orientation. The involution $i$ is thought of as reversing the orientation. The map $p$ forgets the emanating arc. The map $s$ returns the emanating arc in the direction pointing away from the vertex. An edge is by definition an $i$-orbit, hence consist of two arcs. An inner edge is an $i$-orbit both of whose elements are in the image of $s$. A port is by definition an arc in the complement of the image of $s$. The set of ports of a graph is called its interface. The local interface of a vertex $v \in V$ is the set of arcs pointing towards it, formally $i\left(s\left(p^{-1}(v)\right)\right)$. A few examples and pictures are given below, in 3.3 and 4.1.

At first look, this definition may not seem so different from the definition in the introduction, and even a bit more redundant. Its merit is the natural notion of morphism:

3.2. Etale morphisms. An etale morphism of graphs is a commutative diagram

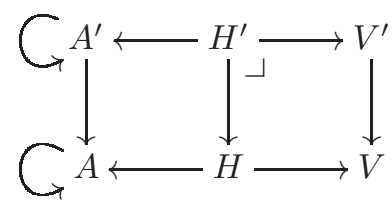

in which the right-hand square is a pullback. The pullback condition says that each vertex must map to a vertex of the same valence. Let 
$\mathbf{G r}$ denote the category of graphs and etale morphisms. (Note that $[\mathbf{9}]$ considers only connected graphs.) An open subgraph (inclusion) is an etale morphism that is injective levelwise. In this note, all subgraphs considered are open, and we suppress the adjective 'open'.

3.3. Unit graph and effective graphs. The unit graph, denoted I, is the graph with one edge and no vertices. It is given by

$$
\mathrm{C}^{2} \longleftarrow 0 \longrightarrow 0
$$

This graph does not have any correspondent in the BM category. In the following we will often have to make exception for it, and more generally exclude graphs with isolated edges (an edge is isolated if neither of its arcs are in the image of $s$ ). A graph is effective if it is nonempty and has no isolated edges. Note that an effective open subgraph is determined by its vertices. Unit graphs are, however, essential for the colimit features of $\mathbf{G r}$, treated next.

3.4. Colimits and monoidal structure. The category $\mathbf{G r}$ has sums (disjoint union of graphs), computed levelwise (i.e. by disjoint union of the sets involved). The empty graph is the neutral object for the symmetric monoidal structure given by sums. The category $\boldsymbol{G r}$ also has other useful colimits, namely certain coequalisers and pushouts over unit graphs, expressing precisely grafting of graphs at their ports. These colimits are also computed levelwise. Precisely, a diagram

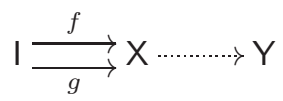

has a colimit if $f$ and $g$ map to different edges in X, such that $f$ maps one of the two arcs in I to a port and $g$ maps the other arc in I to another port: the coequaliser $Y$ is interpreted as the graph obtained by gluing at the two ports. If $\mathrm{X}$ is effective then also $\mathrm{Y}$ is effective.

Similarly, pushouts over a unit graph $\mathbf{X} \stackrel{f}{\longleftarrow} \mathrm{I} \stackrel{g}{\longrightarrow} \mathbf{X}^{\prime}$ exist if the corresponding coequaliser exists $\mathrm{I} \underset{g}{\stackrel{f}{\rightleftarrows}} \mathrm{X}+\mathrm{X}^{\prime} \ldots \ldots \ldots \ldots, \mathrm{Y}$. The possibility of handling gluing formally in terms of colimits, and the fact that these colimit are computed levelwise, is a crucial feature of the category $\mathbf{G r}$.

3.5. Reduced covers. A family of etale morphisms with codomain $X$ is called a covering family of $\mathbf{X}$ if it is jointly surjective on edges and vertices; this defines the etale topology on the category Gr. A covering 
family is called reduced when no member of the family could be omitted or replaced by a proper subgraph without spoiling the joint surjectivity. In the absence of isolated edges, this means jointly bijective on vertices.

Since $\boldsymbol{G r}$ has sums, one may regard a covering family as a single etale morphism, by taking sum of the domains. We shall do this consistently. The single etale morphism obtained from a reduced covering family is called a reduced cover. The quotient morphism of a coequaliser (or pushout) as in 3.4 is always a reduced cover, and every reduced cover can be described as an iterated coequaliser quotient morphism like this. In particular (still in the absence of isolated edges), the reduced covers of a given graph $\mathrm{X}$ form the boolean lattice of subsets of the set of inner edges of $X$.

Proposition 3.6. Let $\boldsymbol{B} \boldsymbol{M}_{\mathrm{gr}} \subset \boldsymbol{B M}$ denote the wide subcategory with only graftings as morphisms, and let $\mathbf{G} \dot{\mathbf{r}}_{\mathrm{rc}} \subset \mathbf{G r}$ denote the wide subcategory whose objects are the graphs without isolated edges, and whose morphisms are the reduced covers. There is a canonical equivalence

$$
\Phi_{1}: \boldsymbol{B} \boldsymbol{M}_{\mathrm{gr}} \stackrel{\sim}{\longrightarrow} \boldsymbol{G} \dot{\boldsymbol{r}}_{\mathrm{rc}} .
$$

Proof: On objects (cf. also $[\mathbf{1}, 15.3]$ ): given a BM graph $(V, F, \partial, j)$, define a JK graph (1) with the same $V$, with $H:=F$ and $A:=F+T$ (the disjoint union of the set of all flags and the set of all tails), with $s$ the sum inclusion and $p:=\partial$. The fixpoint-free involution $i: A \rightarrow A$ is given by $j_{\tau}$ on the fixpoint-free part of $F$, and interchanges each fixed flag in $F$ with its corresponding copy in $T$. The part $T$ plays the role of ports. It is clear that there are no isolated edges. Conversely, given a JK graph without isolated edges, take the same $V$, put $F:=H$, and define an involution $j: F \rightarrow F$ by $j(f)=s^{-1} i s f$ if $f$ is part of an inner edge, and $j(f)=f$ otherwise.

On morphisms: given a grafting $h: \tau \rightarrow \sigma$ between BM graphs, then since $h^{F}: F_{\sigma} \rightarrow F_{\tau}$ is bijective, we can use its inverse to define the component on half-edges between the corresponding JK graphs. The required map on $\operatorname{arcs} A_{\tau} \rightarrow A_{\sigma}$ has to be a map $F_{\tau}+T_{\tau} \rightarrow F_{\sigma}+T_{\sigma}$. For the left-hand square of (2) to commute, we are forced to take $f \mapsto$ $\left(h^{F}\right)^{-1}(f)$ for $f \in F_{\tau}$. For the map to be compatible with the fixpointfree involutions $i$, we are also forced to take $f \mapsto j_{\sigma}\left(h^{F}\right)^{-1}(f)$ for $f \in T_{\tau}$. Conversely, given a reduced cover between JK graphs without isolated edges, since it is bijective on vertices, it is also bijective on half-edges (by the pullback condition), so we can obtain a grafting between the corresponding BM graphs by taking the inverse on flags, and simply forgetting the component on arcs. 


\section{Graphical species, monad, and Kleisli category}

4.1. Elementary graphs. An elementary graph is a connected graph without inner edges. (Connected means: nonempty and not the sum of smaller graphs.) Here are the first few elementary graphs:

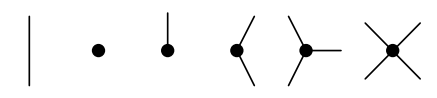

The first one is the unit graph already mentioned; the remaining ones are the corollas $\mathrm{n}$, given by

$$
C^{n}+n^{\prime} \longleftarrow n^{\prime} \longrightarrow 1,
$$

where $n$ and $n^{\prime}$ are finite sets with a bijection $n \stackrel{\sim}{\longrightarrow} n^{\prime}$ defining the involution. (The set of ports of $\mathrm{n}$ is thus $n$.) Let elGr denote the full subcategory of $\mathbf{G r}$ consisting of the elementary graphs.

It is easy to check that every graph $G$ is canonically a colimit in $\mathbf{G r}$ of its elementary subgraphs. The indexing category is the category of elements el $(\mathrm{G}):=\mathbf{e l G r} \downarrow G$. The canonical colimit decomposition of a graph is also a canonical cover, and it follows readily that there is an equivalence of categories between presheaves on $\mathbf{e l G r}$ and sheaves on $\boldsymbol{G r}$, for the etale topology:

$$
\operatorname{PrSh}(\mathrm{elGr}) \stackrel{\sim}{\longrightarrow} \operatorname{Sh}(\mathrm{Gr})
$$

4.2. Graphical species. A presheaf $F: \mathbf{e l G} \boldsymbol{r}^{\circ \mathrm{p}} \rightarrow \boldsymbol{S e t}$ is called a graphical species $[\mathbf{9}]$; its value on $\mathrm{n}$ is denoted $F[\mathrm{n}]$. Explicitly, a graphical species is given by an involutive set $C=F[1]$ (of 'colours'), and for each $n \in \mathbb{N}$ a set $F[\mathrm{n}]$ (of 'operations') with $2 n$ projections to $C$, permuted by a $\mathfrak{S}_{n}$-action on $F[\mathrm{n}]$ and by the involution on $C$.

Each graphical species $F$ defines a notion of $F$-graph: these are graphs decorated on edges by the colours of $F$ and on vertices by the operations of $F$. More formally, the category of $F$-graphs is the comma category $\mathbf{G r} \downarrow F$.

The equivalence $\boldsymbol{P r S h}(\mathbf{e l G r}) \simeq \boldsymbol{S h}(\boldsymbol{G r})$ means that every graphical species can be evaluated not only on elementary graphs but on all graphs: if $F$ is a graphical species and $G$ is a graph, then

$$
F[\mathrm{G}]=\lim _{\mathrm{E} \in \mathrm{el}(\mathrm{G})} F[\mathrm{E}],
$$

where $E$ runs over the category of elements of $G$, i.e. all the elementary subgraphs of $\mathrm{G}$ and the way they are glued together to give $\mathrm{G}$. 


\subsection{The idea of generating more graph morphisms by a monad.} The etale morphisms, and in particular the injective ones, serve as the geometric fabric supporting further algebraic features, essentially graph substitution, which will now be generated by a monad defined in terms of colimits. The new morphisms generated allow for refining a graph by substituting a foreign graph into a vertex. For the notions of monad and Kleisli category we refer to Mac Lane $[\mathbf{1 4}$, Chapter VI].

Generating new morphisms by means of a monad is an important construction in the combinatorics of higher structures [17]. As important examples, the simplex category $\Delta$ arises in this way from the freecategory monad, Joyal's cell category $\Theta$ from the free-strict- $\omega$-category monad $[\mathbf{3}]$, the Moerdijk-Weiss category $\Omega$ of trees from the free-operad monad $[\mathbf{1 1}]$.

The following description of the monad and its restricted Kleisli category is essentially that of Joyal-Kock [9], but with modified conditions imposed on $\mathrm{n}$-graphs. These conditions control what kind of graphs are allowed to be substituted into a vertex, and it is modified in order to accommodate the Borisov-Manin mergers. The adjustments are explained in 4.7 below. Monads of this flavour - defined as a colimit of limits abound in the literature on operads and related structures. The general theory of such monads can be efficiently cast in a polynomial formalism, for which the current state-of-the-art is Batanin-Berger [1] , but going in this direction is beyond the scope of this note.

4.4. $\mathrm{n}$-graphs. Let $n$ be a finite set. An $\mathrm{n}$-graph is a graph $\mathrm{G}$ whose set of ports is $n$ (or more precisely, equipped with a bijection with the ports of $n$ ). A morphism of $n$-graphs is an isomorphism leaving the set of ports fixed (or more precisely, compatible with the specified bijections). We shall need only effective $\mathrm{n}$-graphs and their isomorphisms, forming a groupoid denoted $\mathrm{n}-\boldsymbol{G} \boldsymbol{r}_{\text {iso }}$.

4.5. Monad $[\mathbf{9}]$. Define a monad by the assignment

$$
\begin{aligned}
\operatorname{PrSh}(\text { elGr }) & \longrightarrow \operatorname{PrSh}(\text { elGr }) \\
F & \longmapsto \bar{F},
\end{aligned}
$$

where $\bar{F}$ is the graphical species given by $\bar{F}[I]:=F[I]$ and

$$
\bar{F}[\mathrm{n}]:=\operatorname{colim}_{\mathrm{G} \in \mathrm{n}-\mathrm{Gr} \boldsymbol{r}_{\text {iso }}} F[\mathrm{G}]=\sum_{\mathrm{G} \in \pi_{0}\left(\mathrm{n}-G \boldsymbol{r}_{\text {iso }}\right)} \frac{F[\mathrm{G}]}{\operatorname{Aut}_{\mathrm{n}}(\mathrm{G})}=\pi_{0}\left(\mathrm{n}-\mathbf{G} \boldsymbol{r}_{\text {iso }} \downarrow F\right) .
$$

The monad multiplication is described as follows. $\bar{F}[\mathrm{n}]$ is the set of isomorphism classes of effective $\mathrm{n}-F$-graphs: it is the set of ways to decorate effective n-graphs by the graphical species $F$. Now $\overline{\bar{F}}[\mathrm{n}]$ is the set of 
effective n-graphs decorated by effective $F$-graphs: this means that each vertex is decorated by an $F$-graph with matching interface. We can draw each vertex as a circle with the decorating $F$-graph inside, and the monad multiplication then consists in erasing these circles, turning a graph with vertices decorated by $F$-graphs into a single $F$-graph. More formally, we can use the n-graph as indexing a diagram of $F$-graphs, and then take the colimit in $\mathbf{G r}$. In detail, the groupoid $\mathbf{G r}_{\text {iso }} \downarrow \bar{F}$ has as objects pairs $(\mathrm{R}, \phi)$ where $\mathrm{R}$ is a graph, and $\phi: \operatorname{Hom}(-, \mathrm{R}) \rightarrow \bar{F}$ is a natural transformation. Equivalently we can regard $\phi$ as a functor el(R) $\rightarrow \boldsymbol{e l G} \boldsymbol{r} \downarrow \bar{F}$. Now there is also a canonical functor $\mathbf{e l G} \downarrow \downarrow \bar{F} \rightarrow \boldsymbol{G r} \downarrow F$, which takes unit graphs to unit graphs, and takes a corolla decorated by an $F$-graph to that same $F$-graph. We take the colimit of the composite functor $\mathrm{el}(\mathrm{R}) \rightarrow \boldsymbol{e l G r} \downarrow \bar{F} \rightarrow \boldsymbol{G r} \downarrow F$ to obtain a single $F$-graph. The whole construction defines a functor

$$
G \boldsymbol{r}_{\text {iso }} \downarrow \bar{F} \longrightarrow G \boldsymbol{r}_{\text {iso }} \downarrow F
$$

whose fibrewise $\pi_{0}$ defines the monad multiplication. The unit for the monad interprets an $F$-corolla as an $F$-graph. (More details and a careful proof of associativity can be found in [12], in the case of directed graphs.)

4.6. 'Compact coloured props'. Let $\boldsymbol{C C P}$ denote the category of algebras for the monad $F \mapsto \bar{F}$, tentatively called compact coloured props (although rightly the word 'effective' ought to be part of the name). Hence a compact coloured prop is a graphical species $F: \mathbf{e l G r}{ }^{\mathrm{op}} \rightarrow \boldsymbol{S e t}$ equipped with a structure map $\bar{F} \rightarrow F$ : it amounts to a rule which for any effective $\mathrm{n}$-graph $\mathrm{G} \in \mathrm{n}-\boldsymbol{G r}_{\text {iso }}$ gives a map $F[\mathrm{G}] \rightarrow F[\mathrm{n}]$, i.e. a way of constructing a single operation from a whole graph of them. This rule satisfies an associativity condition (cf. [14, Chapter VI]), amounting roughly to independence of the different ways of breaking the computation into steps.

4.7. Choices involved in the definition of the CCP monad. By adjusting the conditions imposed on n-graphs, one can get different monads. The original choice of Joyal-Kock $[\mathbf{9}]$ is to allow only connected n-graphs. This excludes the empty graph, but allows the unit graph, and leads to a notion of compact symmetric multicategory, essentially a coloured version of the modular operads of Getzler and Kapranov [7].

For the present purposes the connectedness requirement is given up, in order to be general enough to model also prop-like structures, and not just operad-like structures. Specifically it is the availability of nonconnected graphs in $\mathbf{n}-\boldsymbol{G r}_{\text {iso }}$ that leads to morphisms corresponding to the mergers of Borisov-Manin. On the other hand we choose not to allow the empty graph in $\mathbf{0 - G} \boldsymbol{r}_{\text {iso }}$. This choice is merely taken in order 
to agree with Borisov-Manin. Actually the empty graph is a perfectly valid 0 -graph, and it could be included in $\mathbf{0 -} \boldsymbol{G r}_{\text {iso }}$ without problems. It would actually make sense to include it, so as to allow an isolated vertex to be refined into the empty graph, in turn a substitution relevant for the graphical modelling of props, where it accounts for the neutral element in the monoid of $(0,0)$-operations, not guaranteed in the formalisms of [4] or [10]. See also Remark 10.5 of Batanin-Berger [1] for related discussion. Finally we choose to exclude the unit graph I from $2-\mathbf{G r}_{\text {iso }}$, and with it all graphs with isolated edges. A superficial reason is to arrive precisely at the BM category, where unit graphs are not allowed. But a deeper reason is that Proposition 6.1 below would not be true if we allowed I as an object in $2-\mathbf{G r}_{\text {iso }}$.

4.8. Kleisli category. Recall that the Kleisli category (see Mac Lane $[\mathbf{1 4}, \S \mathrm{VI} .5])$ is the full subcategory of the category of algebras spanned by the free algebras. By adjunction, this can also be described as the category whose objects are graphical species, and where a morphism $F \rightarrow Y$ is a morphism of graphical species $F \rightarrow \bar{Y}$. We are interested in the full subcategory $\widetilde{\mathbf{G r}}$ of the Kleisli category given by restricting to graphs, via the fully faithful embedding $\boldsymbol{G r} \rightarrow \boldsymbol{P r S h}(\mathbf{e l G r})$. In [9] this meant restricting to connected graphs (including the unit graph). In the present modified version we allow non-connected graphs, but exclude graphs with isolated edges, such as most importantly the unit graph. We do allow the empty graph as object in the restricted Kleisli category $\widetilde{\mathbf{G r}}$

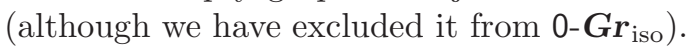

In intuitive terms, a morphism in this restricted Kleisli category $\widetilde{\mathbf{G r}}$ is a map that sends edges to edges and sends vertices to effective subgraphs with the same interface, and respects the flag incidences. More formally, a Kleisli morphism from graph $\mathrm{R}$ to graph $\mathrm{Y}$ is a morphism of graphical species $\mathrm{R} \rightarrow \overline{\mathrm{Y}}$. This can also be described as a functor el $(\mathrm{R}) \rightarrow \boldsymbol{G r} \downarrow \mathrm{Y}$, with the property that each edge element of el(R) is sent to a unit graph (over $\mathrm{Y}$ ) and each $\mathrm{n}$-vertex element $x$ is sent to an effective $\mathrm{n}$-graph $\mathrm{S}_{x}$ (over $\mathrm{Y}$ ). (The vertex condition involves a specific bijection between the interface of the R-corolla at $x$ and the interface of the graph $\mathrm{S}_{x}$.) The colimit of this diagram exists in $\mathbf{G r} \downarrow \mathbf{Y}$, and is denoted $\mathrm{S}$ : it is the graph obtained by gluing together all the graphs $S_{x}$ according to the same 'recipe' el $(\mathrm{R})$ that serves to assemble $\mathrm{R}$ from its elements. The graph $\mathrm{S}$ is etale over $Y$ by construction. (The formal colimit description of the various notions involved has been detailed in [12], in the case of directed graphs.)

There is a subtlety here: for all colimit computations we work in $\mathbf{G r}$ where the unit graph is a crucial element. However, we only glue effective 
graphs, and the result is always effective again, so that both input and output to the computation are objects in $\widehat{\mathbf{G r}}$.

The following lemma is easy, but important.

Lemma 4.9. Every cover morphism $\mathrm{R} \rightarrow \mathrm{R}^{\prime}$ is an epimorphism in the Kleisli category. That is, the extension

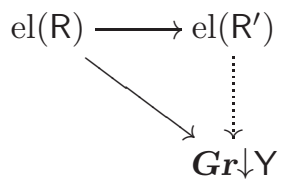

is unique if it exists.

Proof: This follows since the functor el $(R) \rightarrow \mathrm{el}\left(\mathrm{R}^{\prime}\right)$ is surjective on objects, and in particular is an epi-functor.

4.10. Generic morphisms and generic factorisations. We say that a Kleisli morphism $\mathrm{R} \rightarrow \mathrm{Y}$ as above is generic if the colimit graph $\mathrm{S}$ is terminal in $\boldsymbol{G r} \downarrow \mathbf{Y}$, or, equivalently, if the etale morphism $\mathrm{S} \rightarrow \mathrm{Y}$ is invertible. We shall see in a moment that generic morphisms have a natural interpretation as refining the vertices into effective graphs with matching interfaces.

It follows from the previous descriptions that every Kleisli morphism $\mathrm{R} \rightarrow \mathrm{Y}$ has a canonical factorisation into a generic morphism $\mathrm{R} \rightarrow \mathrm{S}$ followed by a free morphism $\mathrm{S} \rightarrow \mathrm{Y}$. 'Free' just means that it is the image of a etale morphism under the monad. Such generic factorisations can be deduced formally from properties of the monad [17]. (In fact, these abstract properties of the monad allows one to characterise the graphs among all graphical species as those objects that can arise as the middle object of a generic factorisation of a Kleisli morphism from a representable (i.e. an elementary graph).)

\section{Generic morphisms (refinements) versus compressions}

5.1. Generic morphisms as graph refinements. From the general description of the Kleisli morphisms, we see that generic morphisms out of a graph $\mathrm{R}$ are given by collections of effective graphs $\mathrm{S}_{x}$ indexed by the vertices of $R$, which are then glued together in the same way as the corollas in $\mathrm{R}$ are glued together to give $\mathrm{R}$. Furthermore, each $\mathrm{S}_{x}$ has the same interface as the corolla at $x$. Geometrically, what the generic 
morphism does is therefore to refine each vertex in $\mathrm{R}$ into an effective graph with the same interface.

\subsection{Duality between generic morphisms and reduced covering} families. Each generic morphism with codomain $S$ gives rise to a reduced covering family of $\mathrm{S}$, namely $\left(\mathrm{S}_{x} \rightarrow \mathrm{S} \mid x \in V\right)$ corresponding to the quotient morphism $\sum_{x} \mathrm{~S}_{x} \rightarrow \mathrm{S}$ in the colimit description (it is reduced since the graphs $\mathrm{S}_{x}$ are effective). Conversely, every reduced covering family $\left(\mathrm{S}_{x} \rightarrow \mathrm{S} \mid x \in V\right)$ is the quotient morphism of a canonical coequaliser diagram of graphs of form

$$
\sum_{e} \mathrm{I} \longmapsto \sum_{x} \mathrm{~S}_{x}
$$

where $e$ runs over the set of edges of $\mathbf{S}$ hit twice by the cover. Since the cover is reduced, no edge is hit more than twice, and all the $S_{x}$ are effective (but not necessarily connected). The same shape of coequaliser diagram can be formed with a corolla $\mathrm{n}_{x}$ in place of each $\mathrm{S}_{x}$ :

$$
\sum_{e} \mathrm{I} \longmapsto \sum_{x} \mathrm{n}_{x} \text {. }
$$

The colimit of this diagram is a graph $\mathrm{R}$ with a vertex for each $x \in V$. The assignment $x \mapsto \mathrm{S}_{x}$ now defines a generic morphism $\mathrm{R} \rightarrow \mathrm{S}$. It is readily seen that these two constructions are essentially inverse to each other. This 'duality' between reduced covering families and generic morphisms was first observed by Berger $[\mathbf{3}]$ in the cases of $\Delta$ and $\Theta$; see $[\mathbf{1 1}$, Remark 2.3.2] for the case of trees.

5.3. Diagrammatic description of generic morphisms. It is a key feature of the JK formalism that etale morphisms are given by straightforward diagrams (2). A similar diagrammatic description is possible for refinement morphisms. Namely, a refinement morphism $\mathrm{R} \rightarrow \mathrm{S}$ is specified unambiguously by a diagram

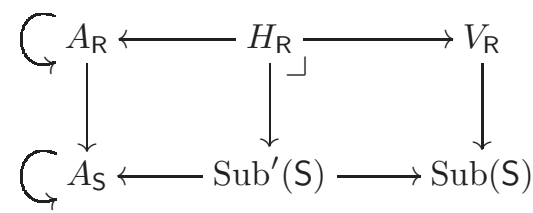

in which the right-hand square is a pullback, and required to be bijective on ports. The bottom diagram is not a graph, only a graphical species. Here $\operatorname{Sub}(S)$ is the set of effective subgraphs of $S$, and $\operatorname{Sub}^{\prime}(S)$ is the set of effective subgraphs of $S$ with a specified outer flag. The outer flags of an (effective) subgraph are $i$ of its ports. Note that applying the involution ensures that the notion agrees with the flags of a vertex 
considered as a one-vertex subgraph, and let us define ports of a diagram like the bottom row as those arcs that are not $s$ of a flag of any effective subgraph. It is clear that the ports of this bottom row are just the ports of S.

Indeed, given a generic morphism, the assignment $x \mapsto \mathrm{S}_{x}$ defines the right-most vertical map. The middle vertical map is canonically defined since each flag belongs to a vertex $x$, and the data in a generic morphism includes bijections between the flags of $x$ and the flags of $\mathrm{S}_{x}$. Commutativity of the left-hand square and with the involutions expresses precisely that the gluing data is the same for $\mathrm{S}$ from the $\mathrm{S}_{x}$ as for $\mathrm{R}$ from its vertex subgraphs. From this it is also clear that the left-hand map induces a bijection on ports. Conversely, given such a diagram, the assignment $x \mapsto \mathrm{S}_{x}$ is already given by the right-hand map, and it has the correct interfaces because the second square is a pullback. It is routine to check that the two constructions are inverse to each other.

Proposition 5.4. Let $\boldsymbol{B M}_{\text {comp }} \subset \boldsymbol{B} \boldsymbol{M}$ denote the wide subcategory with only the compression morphisms, and let $\widetilde{\mathbf{G}} \boldsymbol{r}_{\text {gen }} \subset \widetilde{\mathbf{G r}}$ denote the wide subcategory with only generic morphisms. There is a canonical equivalence

$$
\Phi_{2}: \boldsymbol{B} \boldsymbol{M}_{\mathrm{comp}} \stackrel{\sim}{\longrightarrow} \widetilde{\boldsymbol{G r}}_{\mathrm{gen}}^{\mathrm{op}} .
$$

Proof: We define functors in both directions.

Given a generic morphisms $\mathrm{R} \rightarrow \mathrm{S}$ in terms of a diagram (3), let $\rho$ and $\sigma$ be the corresponding BM graphs, as in the object-part of the proof of Proposition 3.6. We need to construct a compression morphism $h: \sigma \rightarrow \rho$. For each vertex $x \in V_{\mathrm{R}}$, we have an effective subgraph $\mathrm{S}_{x} \subset \mathrm{S}$, and we have a reduced cover $\sum_{x} \mathrm{~S}_{x} \rightarrow \mathrm{S}$. Since it is bijective on vertices, every vertex $v$ of $\mathrm{S}$ belongs to a unique subgraph $\mathrm{S}_{x}$. We define the map $h_{V}: V_{\sigma} \rightarrow V_{\rho}$ by sending $v$ to $x$. It is surjective as required since each subgraph $\mathrm{S}_{x}$ has at least one vertex by the effectivity assumption. Now we define $h^{F}: F_{\rho} \rightarrow F_{\sigma}$ to be the composite $H_{\mathrm{R}} \rightarrow \mathrm{Sub}^{\prime}(\mathrm{S}) \rightarrow H_{\mathrm{S}}$. This map is injective as a consequence of the fact that the graphs $S_{x}$ are effective. Indeed, each flag in $\mathrm{R}$ is either $i$ of a port, in which case its image is too, and generic morphisms are bijective on ports, or it is part of an inner edge of R, which means that it appears once for gluing $\mathrm{R}$ from its elementary graphs, and hence also appears once for gluing together $S$ from the $S_{x}$. Since none of the $S_{x}$ have isolated edges, no step in the gluing procedure can collapse flags. Since a generic morphism induces a bijection on ports, it is also clear that $h^{F}$ is a bijection on tails, and it sends edges to edges (hence verifying (iv)), just by commutativity of the left hand square and the involutions, and 
Axiom (ii), follows also from this. We need to verify Axiom (iii), that endpoints of contracted edges of $h$ have equal image under $h_{V}$. Contracted edges in $\sigma$ is the same thing as inner edges of $\mathrm{S}$ not in the image of R. Let $v, v^{\prime} \in V_{\sigma}$ be the endpoints of a contracted edge of $\sigma$. That the edge is not the image of an inner edge in $\mathrm{R}$ means that $v$ and $v^{\prime}$ belong to the same subgraph $\mathrm{S}_{x}$, and hence they have the same image $x$ under $h_{V}$ as required. Finally, it is clear that $h$ is a compression (so Axiom (ii') is void).

Conversely, given a compression morphism $h: \sigma \rightarrow \rho$ in the BM category, we must construct a diagram like (3). For each $x \in V_{\rho}$, let $\mathrm{S}_{x}$ denote the open subgraph of $\mathrm{S}$ spanned by the set of vertices $h_{V}^{-1}(x)$; it is effective since $h_{V}$ is surjective. The map $h^{F}: F_{\rho} \rightarrow F_{\sigma}$ already gives us $H_{\mathrm{R}} \rightarrow H_{\mathrm{S}}$. For each vertex $x \in V_{\rho}$, the map $h^{F}$ restricts to a bijection $\partial^{-1}(x) \stackrel{\sim}{\longrightarrow} h^{F}\left(F_{\rho}\right) \cap \partial^{-1} h_{V}^{-1}(x)$ (by Lemma 2.5), which tells us on one hand that each flag in $H_{\mathrm{R}}$ incident to $x$ is sent to a flag of the subgraph $\mathrm{S}_{x}$, so as to give altogether a well-defined map $H_{\mathrm{R}} \rightarrow \mathrm{Sub}^{\prime}(\mathrm{S})$, and on the other hand that the right-hand square commutes and is a pullback. Finally we take the map $A_{\mathrm{R}} \rightarrow A_{\mathrm{S}}$ to be

$$
h^{F}+\left.h^{F}\right|_{T_{\rho}}: F_{\rho}+T_{\rho} \longrightarrow F_{\sigma}+T_{\sigma},
$$

well-defined since $h$ is a compression and hence $h^{F}$ preserves tails. The left-hand square now commutes by construction. Since $h^{F}$ also preserves edges (by Lemma 2.4), this map commutes with the involutions. Since $h^{F}$ is bijective on tails, the new map induces a bijection on ports, as required.

It is straightforward to check that the two constructions are inverse to each other.

\section{Borisov-Manin morphisms as cospans in the Kleisli category}

Proposition 6.1. In the restricted Kleisli category $\widetilde{\mathbf{G r}}$, reduced covers and generic morphisms admit pushouts along each other, and the resulting two new morphisms are again a reduced cover and a generic morphism:

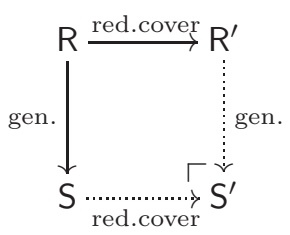


Intuitively, $\mathrm{S}^{\prime}$ is obtained by refining $\mathrm{R}^{\prime}$ by the exact same prescription as $\mathrm{S}$ refines $\mathrm{R}$. This makes sense since $\mathrm{R}^{\prime}$ and $\mathrm{R}$ have the 'same vertices'. Equivalently, $\mathrm{S}^{\prime}$ is obtained by gluing some ports of $\mathrm{S}$ in the same way as $R^{\prime}$ is obtained from $R$. This makes sense since $S$ and $R$ have the 'same ports'. The proof formalises this in terms of colimits:

Proof: Since a reduced cover can be realised as a sequence of quotient morphisms of simple coequaliser diagrams, it is enough to treat pushout along such a morphism, so we assume that the reduced cover $R \rightarrow R^{\prime}$ is the quotient $\mathrm{I}^{\prime} \longrightarrow \mathrm{R} \longrightarrow \mathrm{R}^{\prime}$ expressing the gluing of two ports of $\mathrm{R}$ to form an inner edge of $R^{\prime}$. The generic morphism $R \rightarrow S$ is given by a functor el(R) $\rightarrow \mathbf{G r}$, which in turn unpacks to a coequaliser diagram

$$
\sum_{e} \mathrm{l}_{e} \longrightarrow \sum_{x} \mathrm{~S}_{x} \longrightarrow \mathrm{S},
$$

where $e$ runs over the inner edges of $\mathrm{R}$ and $x$ runs over the vertices of $\mathrm{R}$, and the parallel arrows express the incidences of inner edges and vertices in $R$. The extra gluing producing $R^{\prime}$ from $R$ can now be added to this diagram as an extra summand in the edge sum. This is possible: since that extra gluing connects two distinct port-edges of $\mathrm{R}$ it will also connect two distinct port-edges of $\mathrm{S}$, because a generic morphism is injective on edges and preserves ports. This gives another coequaliser diagram, pictured as the bottom row:

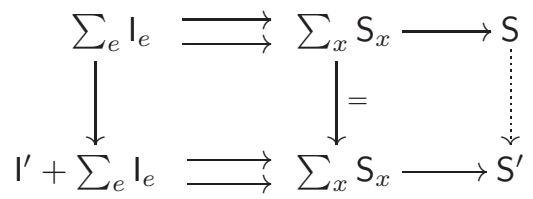

expressing a generic morphism $R^{\prime} \rightarrow S^{\prime}$. The universal property of $S$ as a coequaliser now induces the dotted arrow, which is the reduced cover given precisely by gluing those two ports, i.e. is the quotient morphism $\mathrm{I}^{\prime} \longrightarrow \mathrm{S} \longrightarrow \mathrm{S}^{\prime}$. We now have the promised square of generic and etale morphisms. It commutes because the Kleisli morphism $\mathrm{R} \rightarrow \mathrm{R}^{\prime} \rightarrow$ $\mathrm{S}^{\prime}$ is given by $\mathrm{el}(\mathrm{R}) \rightarrow \boldsymbol{G r} \downarrow \mathrm{S}^{\prime}$ with colimit $\mathrm{S}$ (over $\mathrm{S}^{\prime}$ ) by construction. This shows that the generic-free factorisation is $R \rightarrow S \rightarrow S^{\prime}$ as required. Finally we check that this square is a pullback in the Kleisli category. Given a commutative square of Kleisli morphisms

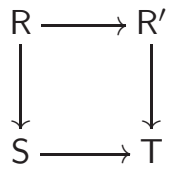


we need to show there is a unique Kleisli morphism $\mathrm{S}^{\prime} \rightarrow \mathrm{T}$ making the triangles commute. There can be at most one extension

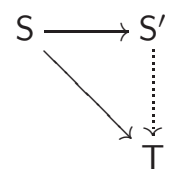

because $\mathrm{S} \rightarrow \mathrm{S}^{\prime}$ is an epimorphism by Lemma 4.9. The graphs $\mathrm{S}$ and $\mathrm{S}^{\prime}$ differ only in that two ports have been glued, so the extension exists if and only if the morphism to $\mathrm{T}$ glues the two ports. But the two ports come from $R$, and via $R \rightarrow R^{\prime} \rightarrow T$ they are certainly glued, so $S^{\prime} \rightarrow T$ is unique as required.

6.2. Remarks. (1) The proposition is analogous to Lemma 2.4.7 in [12], which concerns open inclusions and generic morphisms in the category of acyclic directed graphs. The statement is true more generally for etale morphisms that are injective on vertices, but not for all etale morphisms. It would not be true either if we permitted the unit graph to be substituted into a 2 -valent vertex. Formally this would break an injectivity condition. Essentially, the problem is the following. The substitution of a unit graph into the vertex of a graph consisting of only one vertex and one loop edge ought to yield a loop without vertices. But this is not a valid graph in $\mathbf{G r}$ (and is not included in the Borisov-Manin definition either). This may be seen as a shortcoming of both definitions. References $[\mathbf{1 5}],[\mathbf{1 8}],[\mathbf{8}],[\mathbf{1}]$ address the issue with the vertex-less loop in various ways.

(2) Generic-free pushouts in the simplex category $\Delta$ play an essential role in the theory of decomposition spaces [6] (also called unital 2-Segal spaces $[\mathbf{5}]$ ): a decomposition space is a simplicial space sending genericfree pushouts to pullbacks, precisely the condition ensuring associativity of incidence algebras. The proposition suggests that it might be possible to develop analogous theory for compact coloured props.

6.3. Cospans and factorisation system. In any category in which two classes of morphisms (containing all the isomorphisms) interact under pushouts as in the proposition, one can define a new category with the same objects, whose morphisms are isomorphism classes of cospans whose left leg belongs to one class and the right leg belongs to the other class. Such cospans are composed by taking a pushout. By construction every morphism admits a factorisation as a morphism in the first class followed by a reversed-morphism in the second class. This does not always constitute a factorisation system, since the factorisation is not 
necessarily unique up to unique comparison isomorphism, as required in a factorisation system. However, if one of the two classes consists of epimorphisms, then the comparison isomorphism will be unique.

In the present case, the two classes are the reduced covers and the generic morphisms. Denote by Cosp the category whose objects are graphs without isolated edges, and whose morphisms are iso-classes of cospans in $\widetilde{\mathbf{G r}}$ in which the first leg is a reduced cover and the second leg is a generic morphism

$$
\mathrm{T} \stackrel{\text { red.cover }}{\longrightarrow} \mathrm{S} \stackrel{\text { refinement }}{\longleftarrow} \mathrm{R} .
$$

Since reduced covers are epimorphisms in the Kleisli category (Lemma 4.9), we thus have:

Proposition 6.4. The category Cosp has a factorisation system in which the left-hand class consists of the cospans 'reduced-cover/identityread-backwards' and the right-hand class consists of 'identity/genericmorphisms-backwards'.

Proposition 6.5. The commutation law in $\widetilde{\mathbf{G r}}$ given by pushout is compatible with the commutation law in $\mathbf{B M}$ given by the factorisation system 2.7 via the equivalences $\boldsymbol{B} \boldsymbol{M}_{\mathrm{gr}} \stackrel{\sim}{\longrightarrow} \widetilde{\boldsymbol{G}}_{\mathrm{rc}}$ and $\boldsymbol{B} \boldsymbol{M}_{\mathrm{comp}} \stackrel{\sim}{\longrightarrow} \widetilde{\boldsymbol{G r}}_{\text {gen }}^{\text {op }}$.

Proof: The statement is that given $h$ and $k$ in the commutation square in 2.7, applying the two functors yields a pushout square as in Proposition 6.1. But this is clear from the explicit descriptions given in 2.7 and Proposition 6.1.

Theorem 6.6. The reduced-cover/generic cospan category Cosp is equivalent to the Borisov-Manin category $\boldsymbol{B} \boldsymbol{M}$.

Proof: The argument is a general fact about gluing functors along commutation laws. We already have functors $\Phi_{1}: \boldsymbol{B} \boldsymbol{M}_{\mathrm{gr}} \rightarrow \widetilde{\boldsymbol{G}}_{\mathrm{rc}}$ and $\Phi_{2}$ : $\boldsymbol{B} \boldsymbol{M}_{\text {comp }} \rightarrow \widetilde{\boldsymbol{G r}}_{\text {gen }}^{\text {op }}$ which agree on objects and on isomorphisms. We define a functor $\Phi: \boldsymbol{B M} \rightarrow \boldsymbol{C o s p}$ on morphisms by the assignment

$$
\Phi(h)=\Phi_{2}(c) \circ \Phi_{1}(g)
$$

with reference to the factorisation $h=c \circ g$ of Proposition 2.6:

$$
(\tau \stackrel{g}{\longrightarrow} \sigma \stackrel{c}{\longrightarrow} \rho) \longmapsto\left(\mathrm{T} \stackrel{\Phi_{1}(g)}{\longrightarrow} \mathrm{S} \stackrel{\Phi_{2}(c)}{\longleftrightarrow} \mathrm{R}\right) .
$$

The factorisation $h=c \circ g$ is only determined up to isomorphism, but that is good enough, because the morphisms in Cosp are iso-classes of cospans. This assignment respects composition by Proposition 6.5, hence 
$\Phi$ is a functor. It is clear that $\Phi$ agrees with $\Phi_{1}$ and $\Phi_{2}$ on the subcategories, and it induces an equivalence at the level of maximal groupoids (this follows from either Proposition 3.6 or Proposition 5.4). In particular it is essentially surjective. Finally is follows from Propositions 3.6 and 5.4 that $\Phi$ is fully faithful:

$$
\begin{aligned}
\operatorname{Hom}_{\boldsymbol{B M}}(\tau, \rho) & =\int^{\sigma \in \operatorname{Iso}(\boldsymbol{B M})} \operatorname{Hom}_{\boldsymbol{B} \boldsymbol{M}_{\mathrm{gr}}}(\tau, \sigma) \times \operatorname{Hom}_{\boldsymbol{B} \boldsymbol{M}_{\mathrm{comp}}}(\sigma, \rho) \\
& =\int^{\mathrm{S} \in \mathrm{Iso} \widetilde{\boldsymbol{G r}}} \operatorname{Hom}_{\widetilde{G r}_{\mathrm{rc}}}(\mathrm{T}, \mathrm{S}) \times \operatorname{Hom}_{\widetilde{\boldsymbol{G r}}_{\mathrm{gen}}}(\mathrm{R}, \mathrm{S}) \\
& =\operatorname{Hom}_{\boldsymbol{C o s p}}(\mathrm{T}, \mathrm{R}) .
\end{aligned}
$$

Here the integral signs are coends (see $[\mathbf{1 4}, \S$ IX.6]), which is the appropriate way of summing over all factorisations modulo isomorphisms at the middle object.

6.7. Remark. The BM category of graphs plays an important role in the work of Kaufmann and Ward [10] as source of examples of Feynman categories, an interesting alternative formalism to coloured operads (the two notions have been established equivalent in $[\mathbf{2}]$ ). In that context, the interest is in the full subcategory of the BM category spanned by the disjoint unions of corollas. They show that every such morphism $\tau \rightarrow \rho$ is specified by a so-called ghost graph. Their construction is clarified by the observation that their ghost graph is precisely the graph $\sigma$ appearing in the grafting-compression factorisation, or equivalently, is the apex of the corresponding cospan.

Acknowledgments. I thank Ralph Kaufmann for explaining the Borisov-Manin graph category to me, some years ago. I first met Nils Baas at the IMA in Minneapolis in 2004, and over the years I have learned a lot from many discussions with him on graphs and higher structures. I humbly dedicate this little piece to him at the occasion of his 70th birthday. I regret I could not participate in the birthday conference. This work has been supported by grant number MTM201342178-P of Spain.

\section{References}

[1] M. A. Batanin And C. Berger, Homotopy theory for algebras over polynomial monads, Theory Appl. Categ. 32(6) (2017), $148-253$. 
[2] M. Batanin, J. Kock, And M. Weber, Regular patterns, substitudes, Feynman categories and operads, Theory Appl. Categ. 33(7) (2018), 148-192.

[3] C. Berger, A cellular nerve for higher categories, Adv. Math. 169(1) (2002), 118-175. DOI: 10.1006/aima.2001.2056.

[4] D. V. Borisov And Yu. I. Manin, Generalized operads and their inner cohomomorphisms, in: "Geometry and Dynamics of Groups and Spaces", Progr. Math. 265, Birkhäuser, Basel, 2008, pp. 247-308. DOI : 10.1007/978-3-7643-8608-5_ 4.

[5] T. Dyckerhoff And M. Kapranov, "Higher Segal Spaces I", Lecture Notes in Mathematics (to appear), Springer-Verlag. Preprint (2012): arXiv:1212.3563v1.

[6] I. Gálvez-Carrillo, J. Kock, And A. Tonks, Decomposition spaces, incidence algebras and Möbius inversion I: basic theory, Adv. Math. 331 (2018), 952-1015. DOI: 10.1016/j.aim.2018.03.016.

[7] E. Getzler and M. M. Kapranov, Modular operads, Compositio Math. 110(1) (1998), 65-125. DOI: 10.1023/A:1000245600345.

[8] P. Hackney, M. Robertson, and D. YAu, "Infinity Properads and Infinity Wheeled Properads", Lecture Notes in Mathematics 2147, Springer, Cham, 2015. DOI : 10.1007/978-3-319-20547-2.

[9] A. JoYAl AND J. Kock, Feynman graphs, and nerve theorem for compact symmetric multicategories (extended abstract), Proceedings of the 6th International Workshop on Quantum Physics and Logic (QPL 2009), Special issue of Electron. Notes in Theor. Comput. Sci. 270(2) (2011), 105-113. DOI: 10.1016/j.entcs.2011.01. 025.

[10] R. M. Kaufmann and B. C. Ward, Feynman categories, Astérisque 387 (2017), 161 pp.

[11] J. Kock, Polynomial functors and trees, Int. Math. Res. Not. IMRN 2011(3) (2011), 609-673. DOI: 10.1093/imrn/rnq068.

[12] J. Kock, Graphs, hypergraphs, and properads, Collect. Math. 67(2) (2016), 155-190. DOI: 10.1007/s13348-015-0160-0.

[13] M. Kontsevich and Yu. Manin, Gromov-Witten classes, quantum cohomology, and enumerative geometry, Comm. Math. Phys. 164(3) (1994), 525-562. DOI: 10.1007/BF02101490.

[14] S. Mac LANE, "Categories for the Working Mathematician", Second edition, Graduate Texts in Mathematics 5, Springer-Verlag, New York, 1998. DOI : 10.1007/978-1-4757-4721-8.

[15] M. Markl, S. Merkulov, and S. Shadrin, Wheeled ProPs, graph complexes and the master equation, J. Pure Appl. Algebra 213(4) (2009), 496-535. DOI: 10.1016/j.jpaa.2008.08.007. 
[16] R. Rosebrugh and R. J. Wood, Distributive laws and factorization, Special volume celebrating the 70th birthday of Professor Max Kelly, J. Pure Appl. Algebra 175(1-3) (2002), 327-353. DOI : $10.1016 / \mathrm{S} 0022-4049$ (02) 00140-8.

[17] M. WeBER, Generic morphisms, parametric representations and weakly cartesian monads, Theory Appl. Categ. 13(14) (2004), 191-234.

[18] D. Yau and M. W. Johnson, "A Foundation for PROPs, Algebras, and Modules", Mathematical Surveys and Monographs 203, American Mathematical Society, Providence, RI, 2015. DOI : 10.1090 /surv/203.

Departament de Matemàtiques

Universitat Autònoma de Barcelona

08193 Bellaterra (Barcelona)

E-mail address: kock@mat.uab.cat

Primera versió rebuda el 14 de desembre de 2016, darrera versió rebuda el 10 de gener de 2018. 